\title{
Pseudomonas Cytochrome c Peroxidase. XII. Product
}

\section{Inhibition Studies}

\author{
MARJAANA RÖNNBERG
}

Department of Biochemistry, University of Helsinki, SF-00170 Helsinki 17, Finland

Kinetic studies of the reaction mechanism of Pseudomonas cytochrome $c$ peroxidase (PaCCP) were made by the method of product inhibition using oxidized cytochrome $c$ (551 $P$. aeruginosa) and oxidized Pseudomonas azurin as products. Inhibition by the two oxidized substrates was linearly non-competitive towards the respective reduced electron donor and towards hydrogen peroxide. Although a full kinetic analysis is experimentally impossible in a peroxidase-type reaction, the results do provide some evidence in favour of an ordered reaction mechanism in which hydrogen peroxide is the first to add to PaCCP and electron donor the second.

Pseudomonas cytochrome c peroxidase (PaCCP, EC 1.11.1.5) isolated from $P$. aeruginosa catalyzes the peroxidatic oxidation of the c-type cytochromes and azurin from the same organ. ism.1 The reaction mechanism of PaCCP has been studied by initial velocity techniques and a sequential mechanism with ordered addition of substrates has been proposed for the enzyme.2,3

Alberty and Cleland ${ }^{5}$ have shown product inhibition studies to be useful in determining the mechanistic features of two-substrate reactions, particularly when both the endproducts can be used. Because of the irreversible nature of the peroxidase-type reaction, however, initial velocity as well as product inhibition studies are limited to the oxidation of the electron donor only, and thus neither technique allows a full kinetic analysis of the peroxidase reaction. Nevertheless, product inhibition studies using oxidized electron donors, i.e. oxidized cytochrome c (551 P. aeruginosa) and oxidized azurin, as product inhibitors were undertaken to obtain additional data to confirm the proposed mechanism of the PaCCP. reaction.

The occurrence of a ternary complex in the reaction mechanism was shown by comparing maximal reaction velocities in the presence and absence of a constant concentration of oxidized cytochrome $c$.

As in the earlier studies, ${ }^{2,3}$ all initial velocity measurements were performed in such a way that the phase of initial delay ${ }^{1}$ was avoided, i.e. PaCCP was incubated with the reduced substrates before the reaction was started by addition of hydrogen peroxide.

\section{MATERIALS AND METHODS}

Pseudomonas cytochrome c peroxidase $(P a C C P)$ was prepared from acetone-dried cells of $P$. aeruginosa. ${ }^{6}$ The ratio $A_{407} / A_{280}$ of the preparation was 4.5 and the specific activity was $100-105 \mathrm{U} / \mathrm{mg}$, measured as reported earlier. ${ }^{7}$ The concentration of $\mathrm{PaCCP}$ was deter. mined spectrophotometrically using $A$ (1 \%, $1 \mathrm{~cm}$ ) equal to 12.1 at $280 \mathrm{~nm}$. $^{8}$ The molar concentration of the enzyme was calculated using a molecular weight of $43200 .^{8}$

Yeast cytochrome c peroxidase (YCCP) was prepared as described previously. ${ }^{\circ}$ The ratio $A_{407} / A_{280}$ of the preparation was 1.3. The concentration of the enzyme was determined on the basis of the total hematin content, meas. ured as pyridine ferrohemochrome according to Paul et al.10

Horse heart cytochrome $c$ was a commercial preparation from Sigma Chemical Co., Type III, $98 \%$, used without further purification. The extinction coefficient $\Delta \varepsilon_{550}$ (red.-ox.) $=$ $19.6 \mathrm{mM}^{-1} \mathrm{~cm}^{-1}$ was used for the spectrophotometric determination of the cytochrome concentration. ${ }^{11}$ 
Pseudomonas cytochrome c-551 (Pa-cyt-551) and Pseudomonas azurin were prepared from acetone-dried cells of $P$. aeruginosa by the method of Ambler ${ }^{12}$ and Ambler and Brown. ${ }^{13}$ The purity of the preparation $\left[A_{551}(\right.$ red.) $A_{\text {s70 }}$ (red.) $/ A_{280}$ ] was 1.18 and that of azurin $\left[A_{\text {e28 }}(\right.$ ox. $\left.) / A_{280}\right]$ 0.49. Both azurin and Pa-cyt551 were homogeneous on disc electrophoresis. The concentration of Pa-cyt.551 was determined spectrophotometrically applying the millimolar absorptivity $\Delta \varepsilon_{\mathrm{sb1}}$ (red.-ox.) $=19.0$ $\mathrm{mM}^{-1} \mathrm{~cm}^{-1}$, and the concentration of azurin by using $\varepsilon_{825}($ ox. $)=5.1 \mathrm{mM}^{-1} \mathrm{~cm}^{-1} .^{1}$ Cytochrome was reduced according to Yonetani and Ray ${ }^{14}$ using anaerobic gel filtration on Sephadex G-25. Azurin was reduced with solid sodium dithionite, and excess reductant was removed by exhaustive dialysis against sodium phosphate buffer, pH $6.0, \mu=0.01$. Oxidized cytochrome and azurin were prepared by oxidizing with potassium ferricyanide, and excess oxidant was removed by dialysis against phosphate buffer, $\mathrm{pH} 6.0, \mu=0.01$.

Hydrogen peroxide solutions were prepared from Merck Perhydrol $\left(30 \% \mathrm{H}_{2} \mathrm{O}_{2}\right)$. Peroxide concentration was determined enzymatically with YCCP using horse heart cytochrome $c$ as substrate according to Yonetani..11

Measurements of reaction rates. The activity of PaCCP was assayed spectrophotometrically by measuring the rate of peroxidatic oxidation of fully reduced substrates by the enzyme in sodium phosphate buffer, $\mathrm{pH} 6.0, \mu=0.01$. The reaction was initiated by mixing $10 \mu \mathrm{l}$ of hydrogen peroxide solution with the reaction mixture $(2.0 \mathrm{ml})$ containing varying amounts of reduced cytochrome or azurin and, in inhibition studies, oxidized cytochrome or azurin as well. The reaction was followed by recording the disappearance of the electron donor or the appearance of the product. All velocities were measured on a Cary 15 recording spectrophotometer with the cell compartment thermostated at $25^{\circ} \mathrm{C}$. Reaction mixtures were in. cubated at $25{ }^{\circ} \mathrm{C}$ before the reaction was ini. tiated. Initial velocities were determined from the slopes of the recorded lines and expressed in terms of mol reduced substrate oxidized per mol of enzyme and second.

Disc electrophoresis was carried out in poly. acrylamide gel according to the procedure of Maurer using the basic gel system No. la (pH 8.9, $7 \%$ gel). ${ }^{15}$ The protein bands were stained with Coomassie brilliant blue G.250 (Serva, Heidelberg) by the method of Diezel et al. ${ }^{16}$

Instruments. Spectrophotometric measurements were performed with a Beckman DU-2 spectrophotometer. Enzymatic activities were measured with a Cary 15 recording spectropho. tometer. $\mathrm{pH}$ was measured with a Radiometer pH meter 27 fitted with a combination glass-calomel electrode. Beckman pH 7 buffer No. 3501 was used for standardization.

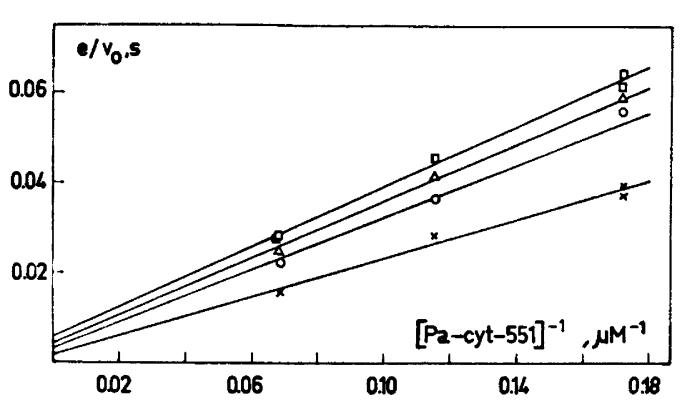

Fig. 1. Product inhibition of Pseudomonas cytochrome c peroxidase by oxidized cytochrome c.551 with reduced cytochrome as the variable substrate. Inhibitor concentrations were $(\mu \mathrm{M})$ : $x, 0 ; 0,8.4 ; \triangle, 14.6 ; \square, 20.8$. Enzyme concentration was $1.6 \mathrm{nM}$ and hydrogen peroxide 81.6 $\mu \mathrm{M}$.

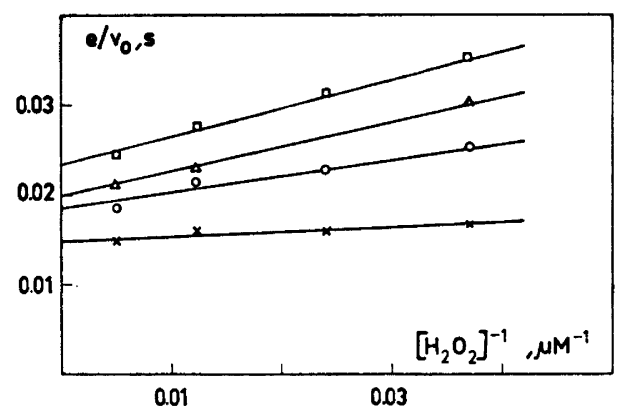

Fig. 2. Product inhibition of PaCCP by oxidized cytochrome c.551 with $\mathrm{H}_{2} \mathrm{O}_{2}$ as the variable substrate. Inhibitor concentrations were $(\mu \mathrm{M}): \times, 0 ; 0,8.4 ; \Delta, 14.6 ; \square, 20.8$. Enzyme concentration was $1.6 \mathrm{nM}$ and reduced cyto. chrome $14.5 \mu \mathrm{M}$.

Reagents were of analytical grade if not otherwise stated.

\section{RESULTS}

The primary Lineweaver-Burk plots appeared to be linear within experimental error in all cases. Inhibition by oxidized Pa-cyt-551 towards hydrogen peroxide was non-competitive in the sense defined by Cleland, ${ }^{5}$ which requires alteration of the slopes and intercepts of the Lineweaver-Burk plots. The inhibition towards reduced $\mathrm{Pa}$-cyt-551 was non-competitive too, although it was difficult to interpret the primary plot of Fig. 1 unambiguously. However,

Acta Chem. Scand. B 30 (1976) No. 8 


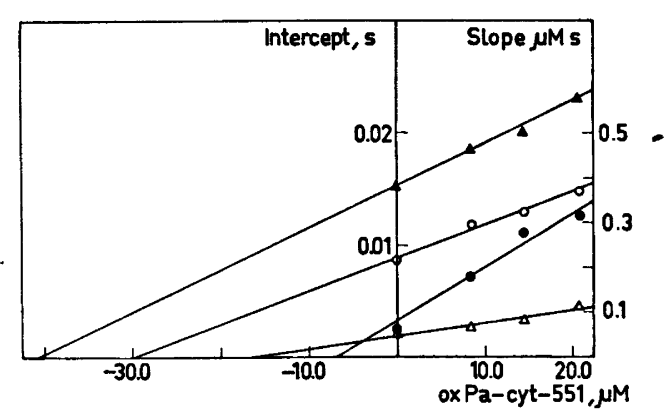

Fig. 3. Replots of slopes $(O, O)$ and intercepts $(\triangle, \Delta)$ of the primary Lineweaver-Burk plots of Figs. 1 and 2 against inhibitor concentration. Open symbols represent data from Fig. 1 and solid symbols slopes and intercepts from Fig. 2. The apparent $K_{i}$ intercept and $K_{i}$ slope-values calculated from the horizontal intercepts of the plot are given in Table 1.

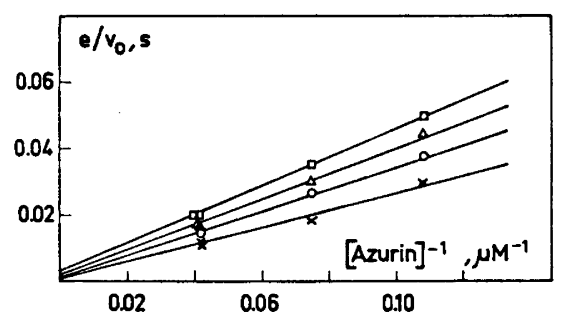

Fig. 4. Product inhibition of PaCCP by oxidized azurin with reduced azurin as the variable substrate. Inhibitor concentrations were $(\mu \mathrm{M}): \times, 0 ; 0,6.6 ; \Delta, 10.6 ; \square, 13.2$. Enzyme concentration was $1.6 \mathrm{nM}$ and hydrogen peroxide $81.6 \mu \mathrm{M}$.

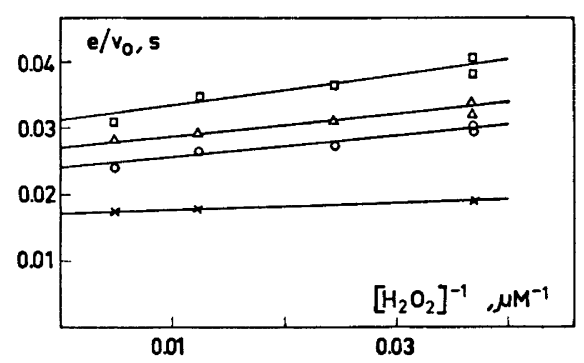

Fig. 5. Product inhibition of PaCCP by oxidized azurin with hydrogen peroxide as the variable substrate. Inhibition concentrations were $(\mu \mathrm{M}): \times, 0 ; 0,6.6 ; \Delta, 10.6 ; \square, 13.2$. Enzyme concentration was $1.6 \mathrm{nM}$ and reduced azurin $13.3 \mu \mathrm{M}$.

Acta Chem. Scand. B 30 (1976) No. 8

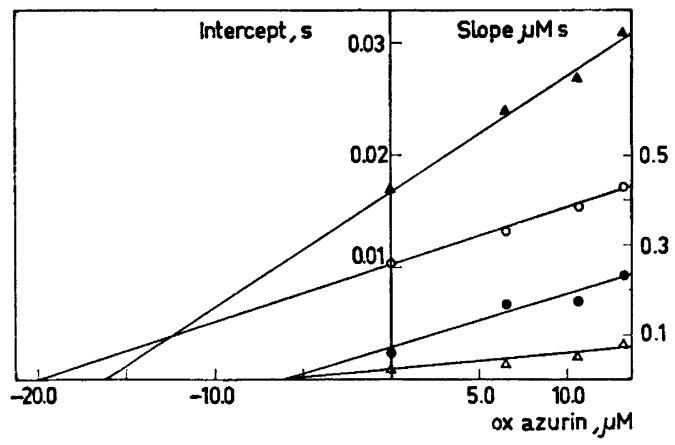

Fig. 6. Replots of slopes $(0,0)$ and intercepts $(\triangle, \Delta)$ of the primary Lineweaver-Burk plots of Figs. 4 and 5 against inhibitor concentration. Open symbols represent slopes and intercepts from Fig. 4 and solid symbols those from Fig. 5. The apparent $K_{\mathrm{ii}}$ and $K_{\text {is }}$-constants calculated from the horizontal intercepts of the plot are given in Table 1.

the Dixon plot, ${ }^{17}$ i.e. the plot of reciprocal initial velocity versus inhibitor concentration, derived from the data of Fig. 1 showed noncompetitive inhibition towards reduced $\mathrm{Pa}$ cyt-551. The Dixon plot from the data of Fig. 2 gave similar results, thus confirming the noncompetitive inhibition by oxidized Pa-cyt-551 towards hydrogen peroxide. The replots of slopes and intercepts of the primary Lineweaver-Burk plots against inhibitor concentration were linear, and from the horizontal intercepts of these plots apparent inhibition constants ( $K_{\mathrm{i} \text { slope }}$ and $K_{\mathrm{i} \text { intercept }}$ ) could be cal. culated. ${ }^{5}$

When oxidized azurin was used as product inhibitor, linear non-competitive inhibition was

Table 1. Inhibition constants for Pseudomonas cytochrome $c$ peroxidase. Type of inhibition: Linear noncompetitive.

\begin{tabular}{lllrl}
\hline Inhibitor & $\begin{array}{l}\text { Variable } \\
\text { substrate }\end{array}$ & $\begin{array}{l}\text { Fixed } \\
\text { substrate }\end{array}$ & $\begin{array}{c}K_{\mathrm{is}} \\
\mu \mathrm{M}\end{array}$ & $\begin{array}{l}K_{\mathrm{ii}} \\
\mu \mathrm{M}\end{array}$ \\
\hline ox Pa-cyt-551 & $\mathrm{Pa-cyt-551}$ & $\mathrm{H}_{2} \mathrm{O}_{2}$ & 30.0 & 16.5 \\
ox Pa-cyt-551 & $\mathrm{H}_{2} \mathrm{O}_{2}$ & $\mathrm{Pa-cyt-551}$ & 7.0 & 40.8 \\
ox azurin & azurin & $\mathrm{H}_{2} \mathrm{O}_{2}$ & 20.0 & 6.9 \\
ox azurin & $\mathrm{H}_{2} \mathrm{O}_{2}$ & azurin & 6.4 & 16.4 \\
\hline
\end{tabular}




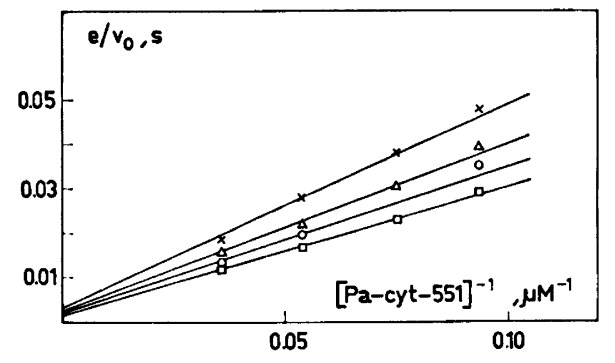

Fig. 7. A double reciprocal plot of initial rate data taken in the presence of $18.4 \mu \mathrm{M}$ oxidized cytochrome c-551 as product inhibitor. Reduced Pa-cyt-551 was the variable substrate. Concentrations of the fixed substrate $\left(\mathrm{H}_{2} \mathrm{O}_{2}\right)$ were $(\mu \mathrm{M}): \times, 7.2 ; \Delta, 14.4 ; 0,28.8$; $\square, 144.0$. Data were taken at $25{ }^{\circ} \mathrm{C}$ and $\mathrm{pH}$ 6.0 in sodium phosphate buffer $\mu=0.01$. The PaCCP-concentration was $1.6 \mathrm{nM}$.

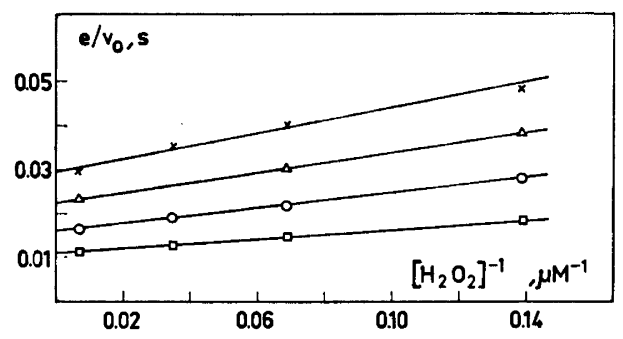

Fig. 8. A double reciprocal plot of initial rate data taken in the presence of $18.4 \mu \mathrm{M}$ oxidized Pa-cyt-551 as product inhibitor. Hydrogen peroxide was the variable substrate. Concentrations of the fixed substrate (red Pa-cyt551) were $(\mu \mathrm{M}): \times, 10.7 ; \Delta, 13.3 ; 0,18.4$; $\square$, 27.8. Other experimental details were as given in the legend to Fig. 7.

observed towards reduced azurin as well as towards hydrogen peroxide. The non-competitive inhibition towards reduced azurin was not immediately apparent, but Dixon plots confirmed non-competitive inhibition for both substrates. Replots of slopes and intercepts of the primary plots versus the concentration of oxidized azurin were linear, and the apparent inhibition constants $\left(K_{\text {is }}\right.$ and $\left.K_{\text {ii }}\right)$ calculated from the plot are given in Table 1 .

Initial velocity measurements in the presence of a constant concentration of oxidized Pa-cyt$551(18.4 \mu \mathrm{M})$ gave linear Lineweaver-Burk plots. The secondary plots of intercepts and

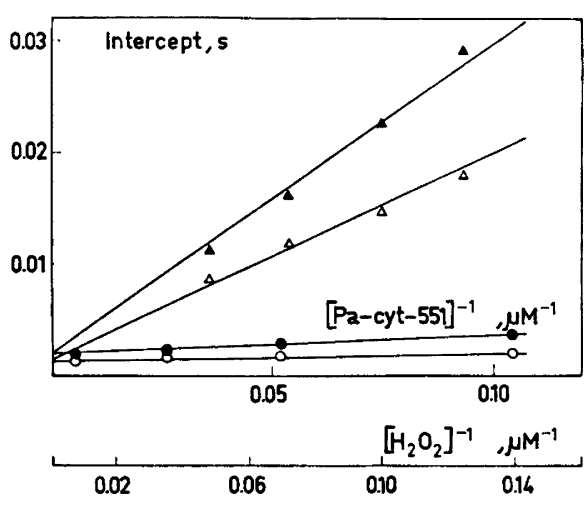

Fig. 9. Secondary plots of primary plot intercepts (see Figs. 7 and 8) versus the reciprocal of fixed substrate concentration. Circles, whether open or solid, represent data in which $\mathrm{H}_{2} \mathrm{O}_{2}$ was present as the fixed substrate. Triangles, whether open or solid, represent data taken with Pa-cyt-551 as the fixed substrate. Open circles or triangles represent data taken in the absence of product inhibitor. Solid circles or triangles represent data taken in the presence of oxidized Pa-cyt-551 (18.4 $\mu \mathrm{M})$ as product inhibitor. Experimental details are given in the legend to Fig. 7.

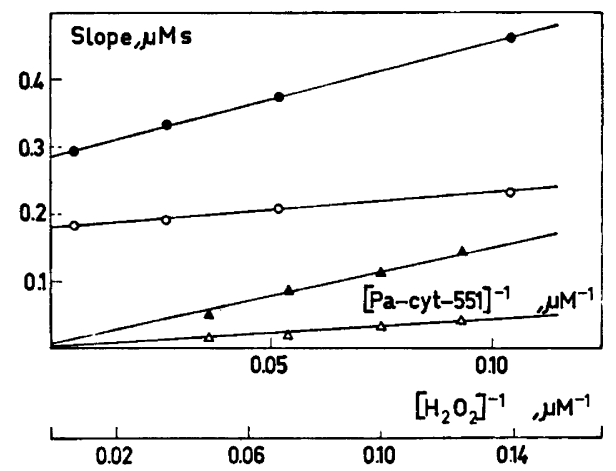

Fig. 10. Secondary plots of primary plot slopes (see Figs. 7 and 8) versus the reciprocal of the fixed substrate concentration. Circles, whether open or solid, represent data taken with $\mathrm{H}_{2} \mathrm{O}_{2}$ as the fixed substrate. Triangles, whether open or solid, represent data taken with Pa-cyt-551 as the fixed substrate. Open circles or triangles represent data taken in the absence of product inhibitor. Solid circles or triangles represent data taken in the presence of oxidized Pa-cyt$551(18.4 \mu \mathrm{M})$ as product inhibitor. Experimental details are given in the legend to Fig. 7. 
slopes are shown in Figs. 9 and 10, respectively, together with data obtained in similar experiments in the absence of the oxidized cytochrome. The results were analyzed using the initial-rate equation of Dalziel ${ }^{18}$

$$
\frac{e}{v_{0}}=\phi_{0}+\frac{\phi_{1}}{\left[\mathrm{~S}_{1}\right]}+\frac{\phi_{2}}{\left[\mathrm{~S}_{2}\right]}+\frac{\phi_{12}}{\left[\mathrm{~S}_{1}\right]\left[\mathrm{S}_{2}\right]}
$$

The kinetic constants $\left(\phi_{0}, \phi_{1}, \phi_{2}, \phi_{12}\right)$ were calculated from the secondary plots of intercepts and slopes. The kinetic constants obtained in the presence of the product inhibitor were increased compared to those of the uninhibited reaction. The reciprocal of the maximal reaction velocity, $\phi_{0}$ calculated from Fig. 9 , was $1.5 \times 10^{-3} \mathrm{~s}$, while that of the inhibited reaction $\phi_{0}^{\prime}$ was $2.2 \times 10^{-3} \mathrm{~s}$. In contrast, the Michaelis constants of hydrogen peroxide and reduced cytochrome c-551 were essentially independent of the presence of the inhibitor.

\section{DISCUSSION}

The product inhibition of the Pseudomonas cytochrome $c$ peroxidase reaction by oxidized cytochrome $c$-551 towards reduced cytochrome has earlier been studied in a simple experiment using one inhibitor concentration only.2 The Lineweaver-Burk plot of this preliminary experiment was interpreted as showing competitive inhibition. The type of inhibition could not be checked in other plots because of the single inhibitor concentration used.

The present study, however, shows that inhibition by oxidized cytochrome and azurin towards their respective reduced substrates as well as towards hydrogen peroxide is noncompetitive. The type of inhibition against reduced cytochrome and azurin cannot be unambiguously derived from the LineweaverBurk plots (Figs. 1, 4), but the occurrence of non-competitive inhibition in both cases has been verified using Dixon plots. ${ }^{17}$

Some qualitative conclusions can be drawn from the results even though only one product is available for inhibition studies and the kinetic analysis is thus incomplete. ${ }^{5}$ On the basis of the intersecting initial velocity patterns, it has been suggested that the PaCCP. reaction follows a sequential reaction mechanism. ${ }^{2,3}$ The present product inhibition study helps further to rule out a ping-pong mech- anism, since such a mechanism would require competitive inhibition by oxidized cytochrome towards hydrogen peroxide, which is not the case.

The mechanisms to be more thoroughly considered are Ordered $\mathrm{Bi} \mathrm{Bi,}{ }^{19}$ Rapid Equilibrium Random $\mathrm{Bi} \mathrm{Bi},{ }^{19}$ and the Theorell-Chance mechanism. ${ }^{20}$ In a random mechanism, inhibition by one end-product against either substrate should be competitive, ${ }^{5}$ and so this mechanism is not proposed for PaCCP. On the other hand, the non-competitive inhibition patterns against both reduced cytochrome (or azurin) and hydrogen peroxide are in accordance with an Ordered $\mathrm{Bi}$ Bi mechanism ${ }^{\circ}$ in which cytochrome is the second substrate to add to the enzyme and oxidized cytochrome the first product to leave. If reduced cytochrome were the first substrate, and thus oxidized cyto. chrome the second product, inhibition should be competitive against reduced cytochrome and non-competitive against hydrogen peroxide. The data obtained do not support this sequence, and furthermore, such an order of substrate addition would make PaCCP unique among peroxidases.

According to Alberty, the product inhibition method is capable of detecting ternary complexes of even very short life-times. Consequently, a distinction between an Ordered $\mathrm{Bi} \mathrm{Bi}$ and Theorell-Chance mechanism can be made in experiments in which the product of the second substrate is added initially. In either mechanism adding the product of the first substrate initially has no effect on the maximal velocity. In an ordered ternary complex mechanism the effect of the product inhibitor on the maximum velocity of the reaction reveals that it is the product of the second substrate.

Non-competitive inhibition in itself implies a reduced maximal velocity in the presence of the inhibitor. Moreover, a full two-substrate kinetic study in the presence of a constant concentration of oxidized cytochrome $c .551$ showed a decreased maximal velocity compared to that of the uninhibited reaction (Fig. 9), which confirms the occurrence of a ternary complex in the PaCCP-reaction. A similar experimental arrangement has earlier been used to verify a ternary complex in the reaction of pig heart malic dehydrogenase. ${ }^{21}$ 
The results of the product inhibition studies seem to support an ordered reaction mechanism in which hydrogen peroxide adds to the peroxidase as the first substrate and electron donor as the second.

\section{REFERENCES}

1. Soininen, R. and Ellfolk, N. Acta Chem. Scand. 26 (1972) 861.

2. Ellfolk, N., Rönnberg, M. and Soininen, R. Acta Chem. Scand. 27 (1973) 2171.

3. Rönnberg, M. and Ellfolk, N. Acta Chem. Scand. B 29 (1975) 719.

4. Alberty, R. A. J. Am. Chem. Soc. 80 (1958) 1777.

5. Cleland, W. W. Biochim. Biophys. Acta 67 (1963) 173.

6. Ellfolk, N. and Soininen, R. Acta Chem. Scand. 24 (1970) 2126.

7. Soininen, R. and Ellfolk, N. Acta Chem. Scand. 27 (1973) 35.

8. Ellfolk, N. and Soininen, R. Acta Chem. Scand. 25 (1971) 1535.

9. Ellfolk, N. Acta Chem. Scand. 21 (1967) 175.

10. Paul, K.-G., Theorell, H. and Ákeson, A. Acta Chem. Scand. 7 (1953) 1284.

11. Yonetani, T. J. Biol. Chem. 240 (1965) 4509.

12. Ambler, R. P. Biochem. J. 89 (1963) 341.

13. Ambler, R. P. and Brown, L. H. Biochem. J. 104 (1967) 784.

14. Yonetani, T. and Ray, G. S. J. Biol. Chem. 240 (1965) 3392.

15. Maurer, H. R. Disk-Elektrophorese, Walter De Gruyter, Berlin 1968.

16. Diezel, W., Kopperschläger, G. and Hofmann, E. Anal. Biochem. 48 (1972) 617.

17. Dixon, M. Biochem. J. 55 (1953) 170.

18. Dalziel, K. Acta Chem. Scand. 11 (1957) 1706.

19. Cleland, W. W. Biochim. Biophys. Acta 67 (1963) 104.

20. Theorell, H. and Chance, B. Acta Chem. Scand. 5 (1951) 1127.

21. Raval, D. N. and Wolfe, R. G. Biochemistry 1 (1962) 1112.

Received January 23, 1976. 